\title{
Opposite Prognostic Impact of Single PTEN-loss and PIK3CA Mutations in Early High-risk Breast Cancer
}

\author{
GEORGIOS LAZARIDIS ${ }^{1 *}$, VASSILIKI KOTOULA ${ }^{2,3 *}$, ELENI VRETTOU ${ }^{2}$, IOANNIS KOSTOPOULOS $^{2}$, \\ KYRIAKI MANOUSOU ${ }^{4}$, KYRIAKI PAPADOPOULOU ${ }^{3}$, ELENI GIANNOULATOU ${ }^{5,6}$, MATTHEOS BOBOS $^{3}$, \\ MARIA SOTIROPOULOU ${ }^{7}$, GEORGE PENTHEROUDAKIS ${ }^{8}$, IOANNIS EFSTRATIOU ${ }^{2}$, \\ ALEXANDRA PAPOUDOU-BAI ${ }^{9}$, AMANDA PSYRRI $^{10}$, CHRISTOS CHRISTODOULOU $^{11}$, \\ HELEN GOGAS $^{12}$, ANGELOS KOUTRAS ${ }^{13}$, ELENI TIMOTHEADOU ${ }^{1}$, \\ DIMITRIOS PECTASIDES ${ }^{14}$, FLORA ZAGOURI ${ }^{15}$ and GEORGE FOUNTZILAS ${ }^{3,16}$ \\ ${ }^{1}$ Department of Medical Oncology, Faculty of Medicine Papageorgiou Hospital, \\ Aristotle University of Thessaloniki, School of Health Sciences, Thessaloniki, Greece; \\ ${ }^{2}$ Department of Pathology, School of Health Sciences, Faculty of Medicine, \\ Aristotle University of Thessaloniki, Thessaloniki, Greece; \\ ${ }^{3}$ Laboratory of Molecular Oncology, Hellenic Foundation for Cancer Research, \\ Aristotle University of Thessaloniki, Thessaloniki, Greece; \\ ${ }^{4}$ Section of Biostatistics, Hellenic Cooperative Oncology Group, Data Office, Athens, Greece; \\ ${ }^{5}$ Victor Chang Cardiac Research Institute, Darlinghurst, NSW, Australia; \\ ${ }^{6}$ The University of New South Wales, Kensington, NSW, Australia; \\ ${ }^{7}$ Department of Pathology, Alexandra Hospital, Athens, Greece; \\ ${ }^{8}$ Department of Medical Oncology, Ioannina University Hospital, Ioannina, Greece; \\ ${ }^{9}$ Department of Pathology, Ioannina University Hospital, Ioannina, Greece; \\ ${ }^{10}$ Division of Oncology, Second Department of Internal Medicine, Attikon University Hospital, Athens, Greece; \\ ${ }^{11}$ Second Department of Medical Oncology, Metropolitan Hospital, Piraeus, Greece; \\ ${ }^{12}$ First Department of Medicine, Laiko General Hospital, \\ National and Kapodistrian University of Athens School of Medicine, Athens, Greece; \\ ${ }^{13}$ Division of Oncology, Department of Medicine, University Hospital, \\ University of Patras Medical School, Patras, Greece; \\ ${ }^{14}$ Oncology Section, Second Department of Internal Medicine, Hippokration Hospital, Athens, Greece; \\ ${ }^{15}$ Department of Clinical Therapeutics, Alexandra Hospital, \\ National and Kapodistrian University of Athens School of Medicine, Athens, Greece; \\ ${ }^{16}$ Aristotle University of Thessaloniki, Thessaloniki, Greece
}

\begin{abstract}
Background/Aim: PTEN-loss and PIK3CA mutations have been addressed as markers of PI3K activation in breast cancer. We evaluated these markers in early high-risk breast cancer (EBC) focusing on PTEN immunohistochemistry
\end{abstract}

This article is freely accessible online.

*The first two Authors contributed equally to this work.

Correspondence to: Georgios Lazaridis, MD, Department of Medical Oncology, Papageorgiou Hospital, Ring Road, Nea Efkarpia, 56 403, Thessaloniki, Greece. E-mail: georlaz@yahoo.gr

Key Words: PTEN immunohistochemistry, heterogeneity, discordance, PI3K pathway, HER2-positive breast cancer, adjuvant chemotherapy, trastuzumab.
(IHC) issues, particularly in HER2-positive disease. Materials and Methods: We examined PTEN-loss and PIK3CA mutations in 1265 EBC patients treated with adjuvant chemotherapy within two clinical trials. Two different methods for the evaluation of PTEN IHC were used, one upfront binary (loss; no-loss) and the other initially multi-scale allowing for the classification of "grey zone" tumors with low and very low PTEN protein expression. Results: PTEN-loss (33.4\% and $22.1 \%$, depending on the IHC method) and PIK3CA mutations (29.6\%) were associated with ER/PgR/HER2-negative and $E R / P g R$-positive disease, respectively. Concordance of the two IHC methods was moderate (Cohen's kappa 0.624). PTEN-loss discrepancy and intra-tumor heterogeneity concerned "grey zone" tumors that were prevalent among HER2-positive cancers. PTEN-loss independently conferred higher risk for relapse and death. Compared to single PIK3CA mutations, 
single PTEN-loss was independently associated with increased risk for relapse and death. Depending on the evaluation method, in HER2-positive cancer, PTEN-loss was without- or of marginal unfavorable prognostic significance. Conclusion: In EBC, PTEN-loss is an independent predictor of poor outcome. When occurring singly, PTEN-loss and PIK3CA mutations have opposite prognostic impact. In HER2-positive disease, assessment of PTEN-loss by IHC appears unreliable and the marker is without clear prognostic significance.

The PI3K/PTEN/AKT axis operates within the PI3K/ $\mathrm{AKT} / \mathrm{mTOR}$ pathway as a key regulator of most cancer hallmarks. In this axis, PTEN (phosphatase and tensin homolog; gene on chromosome 10q23) is a tumor suppressor that dephosphorylates phosphatidylinositol-3,4,5-triphosphate into 4,5-biphosphate and thus deactivates the phosphatidylinositide3-kinase (PI3K) [reviewed in (1-3)]. PTEN-loss and mutations in PIK3CA, the p110 catalytic subunit of PI3K, are linked to the activation of the PI3K pathway, which is targeted by diverse classes of specific and general inhibitors.

PTEN-loss in cancer occurs due to mutation, deletion or transcriptional inactivation (4). In breast cancer, PTEN-loss is infrequently based on PTEN genomic alterations (5), while it appears that decreased expression even without complete loss of this protein may be important for the biology of disease (6-8). The prevalence of PTEN-loss ranges from $4 \%$ to $63 \%$ across different studies, being more frequent in ER/PgR-negative and HER2-negative tumors (9-13). PTENloss has been associated with aggravating clinicopathological features $(13,14)$ and adverse prognosis in all stages of breast cancer (15). In patients with early HER2-positive disease who were treated with adjuvant chemotherapy in the pretrastuzumab era, PTEN-loss has been reported as an adverse prognosticator (11) or without prognostic significance (16); in patients who received adjuvant trastuzumab, PTEN-loss had no clear prognostic significance in the BCIRG-006 trial (11) and in the NCCTG N9831 trial (17). As yet, inhibition of the PI3K pathway has been more successful in ERpositive/HER2-negative [reviewed in $(1,18,19)$ ], but not in HER2-positive disease, despite initial evidence linking PTEN-loss with intrinsic resistance to HER2-inhibition (20).

The objective of this study was to investigate the impact of PTEN-loss, individually and in combination with the PIK3CA mutational status on the outcome of patients with operable breast cancer who had received anthracyclinetaxane-based adjuvant chemotherapy. Although PTEN-loss and no-loss are usually evaluated with immunohistochemistry (IHC), and despite efforts to standardize this method with respect to the biological implications of decreased PTEN protein expression levels (11), there is currently no standard method for the evaluation of this marker. This appears to be a major cause of inconsistent results with respect to PTENloss as a marker in breast cancer $(13,15,17,21,22)$. To address this question, we applied two approaches for the evaluation of PTEN IHC and present the corresponding sets of results for comparison, focusing on HER2-positive disease.

\section{Materials and Methods}

PTEN status was examined in routinely processed tumor tissues (FFPE) from patients with operable breast cancer who had been treated with adjuvant anthracycline-taxane-based chemotherapy within the HE 10/05 (ACTRN-12610000151033) (23) and HE 10/08 (ACTRN-12615000161527) clinical trials by the Hellenic Cooperative Oncology Group (HeCOG) described in (24). Patients with HER2-positive and ER/PgR-positive disease had received trastuzumab and hormonotherapy, respectively, based on local pathology typing. All patients had submitted informed consent for the use of their biological material for research purposes. The study was approved by the Bioethics Committee of the Aristotle University of Thessaloniki School of Health Sciences, Faculty of Medicine (\#77/10June2014) and by the Institutional Review Board of the Papageorgiou Hospital of Thessaloniki (\#725/10May2013). The status of PIK3CA mutations and their impact on patient outcome were previously described for these patients $(24,25)$. All tumors were re-assessed centrally at the Laboratory of Molecular Oncology (MOL; Hellenic Foundation for Cancer Research/ $\mathrm{HeCOG}$ /Aristotle University of Thessaloniki, Thessaloniki, Greece) for histology; construction of low-density tumor microarrays including $2 \times 1 \mathrm{~mm}$ cores per tumor; assessment of the density per stromal area of tumor infiltrating lymphocytes (TILs) according to (26); construction of low-density tissue microarrays including at least $2 \times 1 \mathrm{~mm}$ cores per tumor; clinical subtyping with IHC4 (ER/PgR/HER2/Ki-67) resulting in the 5 classical subtypes described in Table I, with Ki67 cut-off at 14\% for distinguishing between Luminal A and B tumors (27); and, implementation of PTEN IHC. Local/central pathology typing was $88 \%$ concordant for $\mathrm{ER} / \mathrm{PgR}$ and $88.6 \%$ for HER 2 status. For this study, the central annotation of breast cancer subtypes was used.

PTEN IHC. IHC for PTEN protein (clone 6H2.1, DAKO, Glostrup, Denmark) was applied on $3 \mu \mathrm{m}$-thick TMA sections. Following deparaffinization and rehydration, IHC staining was performed in a Bond-Max ${ }^{\mathrm{TM}}$ autostainer (Leica Microsystems, Wetzlar, Germany) using Bond Polymer Refine detection kit (DS9800, Leica). The slides were incubated for 20 min with EDTA (pH 9.0) for antigen unmasking, and for one hour with the primary antibody (dilution 1:300).

PTEN stained TMA sections were centrally and simultaneously evaluated by two breast cancer expert pathologists (E.V., I.K.) for the intensity of staining (negative $=0$; weak $=1+$, intermediate $=2+$, strong $=3+$ ) in stromal and tumor cells. Simultaneous observation was necessary for eliminating the issue of inter-observer variability when scoring low intensity staining. Percentage values were recorded for all intensity levels separately for each core, blinded to the identity of each tumor. Tumors completely negative for PTEN without evaluable stroma or with PTEN negative stroma were excluded from the analysis. We finally evaluated 1257 tumors (Figure 1), and recorded values separately for each core. We applied two approaches for the evaluation of PTEN protein status:

Binary Approach A: Tumor cores were considered as PTEN-noloss, if PTEN protein was expressed in the cytoplasm and/or nucleus of at least $10 \%$ of the tumor cells at intensities $2+$ and $3+$. For tumors with weak stromal staining, PTEN-no-loss was considered 
Table I. Selected patient and tumor characteristics, in the entire cohort and by trial.

\begin{tabular}{|c|c|c|c|c|}
\hline & Entire Cohort $(\mathrm{N}=1265)$ & HE1005 (N=651) & HE1008 $(\mathrm{N}=614)$ & p-Value \\
\hline \multicolumn{5}{|l|}{ Age } \\
\hline$\leq 50$ & $514(40.6 \%)$ & $273(41.9)$ & $241(39.3)$ & \multirow[t]{2}{*}{0.331} \\
\hline$>50$ & $751(59.4 \%)$ & $378(58.1)$ & $373(60.7)$ & \\
\hline \multicolumn{5}{|l|}{ Menopausal status } \\
\hline Postmenopausal & $705(55.8 \%)$ & $341(52.4)$ & $364(59.3)$ & \multirow[t]{2}{*}{0.014} \\
\hline Premenopausal & $560(44.2 \%)$ & $310(47.6)$ & $250(40.7)$ & \\
\hline \multicolumn{5}{|l|}{ Breast surgery } \\
\hline Modified radical mastectomy & $644(51 \%)$ & $331(50.8)$ & $313(51.0)$ & \multirow[t]{2}{*}{0.962} \\
\hline Breast conserving surgery & $621(49 \%)$ & $320(49.2)$ & $301(49.0)$ & \\
\hline \multicolumn{5}{|l|}{ ERPgR status $\#$} \\
\hline Positive & $1066(84.2 \%)$ & $556(85.4)$ & $510(84.2)$ & \multirow[t]{3}{*}{0.538} \\
\hline Negative & $191(15 \%)$ & $95(14.6)$ & $96(15.8)$ & \\
\hline Unknown & $8(0.6 \%)$ & & & \\
\hline \multicolumn{5}{|l|}{ HER2 status } \\
\hline Negative & $999(79 \%)$ & $488(75.0)$ & $511(84.0)$ & \multirow{3}{*}{$<0.001$} \\
\hline Positive & $260(20.6 \%)$ & $163(25.0)$ & $97(16.0)$ & \\
\hline Unknown & $6(0.4 \%)$ & & & \\
\hline \multicolumn{5}{|l|}{ Number of positive nodes } \\
\hline $0-3$ & $871(68.8 \%)$ & $434(66.7)$ & $437(71.2)$ & \multirow[t]{2}{*}{0.084} \\
\hline$\geq 4$ & $394(31.2 \%)$ & $217(33.3)$ & $177(28.8)$ & \\
\hline \multicolumn{5}{|l|}{ Tumor size } \\
\hline$\leq 2$ & $516(40.8 \%)$ & $268(41.2)$ & $248(40.4)$ & \multirow{3}{*}{0.961} \\
\hline $2-5$ & $651(51.4 \%)$ & $333(51.2)$ & $318(51.8)$ & \\
\hline$>5$ & $98(7.8 \%)$ & $50(7.7)$ & $48(7.8)$ & \\
\hline \multicolumn{5}{|l|}{ Histological grade } \\
\hline I & $94(7.4 \%)$ & $45(6.9)$ & $49(8.1)$ & \multirow{4}{*}{0.696} \\
\hline II & $576(45.6 \%)$ & $297(45.6)$ & $279(46.0)$ & \\
\hline III & $588(46.4 \%)$ & $309(47.5)$ & $279(46.0)$ & \\
\hline Unknown & $7(0.6 \%)$ & & & \\
\hline Histological type & & & & \\
\hline Invasive ductal NST* & $821(65 \%)$ & $400(86.0)$ & $421(84.9)$ & 0.081 \\
\hline Invasive lobular & $78(6.2 \%)$ & $30(6.5)$ & $48(9.7)$ & \\
\hline Mixed & $27(2.2 \%)$ & $18(3.9)$ & $9(1.8)$ & \\
\hline Other & $35(2.8 \%)$ & $17(3.7)$ & $18(3.6)$ & \\
\hline Unknown & $304(24 \%)$ & & & \\
\hline Adjuvant hormonotherapy & & & & \\
\hline Yes & $1005(79.4 \%)$ & $521(80.0)$ & $484(79.0)$ & 0.636 \\
\hline No & $259(20.4 \%)$ & $130(20.0)$ & $129(21.0)$ & \\
\hline Unknown & $1(0 \%)$ & & & \\
\hline Adjuvant radiotherapy & & & & \\
\hline Yes & $937(74 \%)$ & $488(75.0)$ & $449(76.0)$ & 0.679 \\
\hline No & $305(24.2 \%)$ & $163(25.0)$ & $142(24.0)$ & \\
\hline Unknown & $23(1.8 \%)$ & & & \\
\hline Herceptin & & & & \\
\hline Yes & $288(22.8 \%)$ & $158(24.3)$ & $130(21.2)$ & 0.189 \\
\hline No & $977(77.2 \%)$ & $493(75.7)$ & $484(78.8)$ & \\
\hline Subtypes & & & & \\
\hline HER2-Enriched & $84(6.6 \%)$ & $51(7.9)$ & $33(5.9)$ & $<0.001$ \\
\hline Luminal A & $494(39 \%)$ & $314(48.8)$ & $180(32.0)$ & \\
\hline Luminal B & $346(27.4 \%)$ & $123(19.1)$ & $223(39.6)$ & \\
\hline Luminal-HER2 & $176(14 \%)$ & $112(17.4)$ & $64(11.4)$ & \\
\hline TNBC & $107(8.4 \%)$ & $44(6.8)$ & $63(11.2)$ & \\
\hline Unknown & $58(4.6 \%)$ & & & \\
\hline Ki67 labeling & & & & \\
\hline $\mathrm{N}$ & 1237 & 641 & 596 & $<0.001$ \\
\hline Median & 20 & 11 & 30 & \\
\hline Range & $0-100$ & $0-100$ & $0-100$ & \\
\hline TILs & & & & \\
\hline $\mathrm{N}$ & 1265 & 651 & 614 & 0.043 \\
\hline Median & 5 & 5 & 5 & \\
\hline Range & $1-90$ & $1-90$ & $1-90$ & \\
\hline
\end{tabular}

\#ER+ 1008/1256 informative (80.3\%), PgR+ 894/1251 informative (71.5\%), ER+/PgR- 168/1249 informative (13.4\%), ER-/PgR+ 56/1249 (4\%); *non-specific type. 
if more than $10 \%$ of tumor cells demonstrated intensity $1+$. This classification, previously used by our Group (16), yielded a binary result for PTEN protein expression, loss and no-loss; here, the "loss" category in fact included completely negative and PTEN-low tumors.

Multi-scale Approach B: A previously described detailed evaluation for PTEN staining was also applied (11). This approach yielded a 5-scale classification for tumor PTEN protein expression: completely negative $=0$; weaker than the weak stroma (very low PTEN expression $)=1 *$; weak (low PTEN expression $)=1+$; intermediate $=2+$; and, strong $=3+$. Categories $1+, 2+, 3+$ were classified as PTEN-no-loss.

Intra-tumor heterogeneity of PTEN protein expression was assessed in 923 tumors with preserved multiple cores. With the binary Approach A, tumors with a heterogeneous core evaluation, i.e., loss and no-loss in the available cores [123 out of 924 tumors evaluable for heterogeneity (13.4\%)] were classified as PTEN noloss. With multi-scale Approach B, tumors with a heterogeneous core evaluation, i.e., $0 \mathrm{vs} .1+, 2+$, or $3+[77$ out of 923 tumors evaluable for heterogeneity $(8.3 \%)$ ] were classified as PTEN-loss. Category $1 *$ was finally classified as PTEN no-loss.

Characteristic examples of PTEN IHC are shown in Figure 2A. As per the manufacturer, the antibody used detects PTEN protein in the cytoplasm and in the nucleus. Although cytoplasmic and nuclear PTEN may have different functions (2), in practice, on paraffin tissue sections, it is impossible to appropriately assess PTEN protein staining separately in the two cellular compartments.

For readability purposes, we present significant results with the binary Approach A in the main text and those with the multi-scale Approach B in the supplementary data (https://figshare.com/s/ c10051be3947b26052fb).

PTEN and PIK3CA genotypes. PTEN amplicons covering the most frequently mutated regions of this gene based on COSMIC and on previous reports $(4,5)$ were included in the previously published panel used for targeted next generation sequencing (NGS) for these tumors (28): ex1, 89624138-89624312; 89624218-89624385; ex7, 89717547-89717720; 89717672-89717822; ex8, 8972076189720934; 89720839-89721012; ex9, 89724991-89725119 (GRCh37 coordinates on chromosome 10). The method, the eligibility criteria, and the PIK3CA mutations (exons 9 and 20) obtained using the same panel have been previously described for an extended series including the present cohort (24). PTEN and PIK3CA genotypes were informative in 961 tumors (Figure 1). We also compared the variant allelic frequencies (VAFs) of the identified mutations, as a surrogate measure of their clonal [VAFs $>25 \%$ (29)] presence in tumors.

Statistical analysis. Patients with unknown PTEN by IHC $(\mathrm{N}=8)$ were classified as PTEN-loss if PTEN was mutant. The derived PTEN status (Figure 1) corresponded to PTEN-loss by IHC and/or PTEN mutant; and, PTEN-no-loss by IHC only.

Disease-free (DFS) and overall survival (OS) were defined as the time (in months) from the date of diagnosis until event (progression or death from any cause) or, in the absence of event, until date of last contact. Deaths without prior registered progression were considered as events. The clinicopathological parameters studied in association with DFS/OS are shown in Table I. The study variables were: PIK3CA mutations; PTEN IHC; PTEN status; and, PTEN status/PIK3CA mutations. For multivariate analyses, we considered clinicopathological parameters (univariate cox $p<0.10$ ), centrally

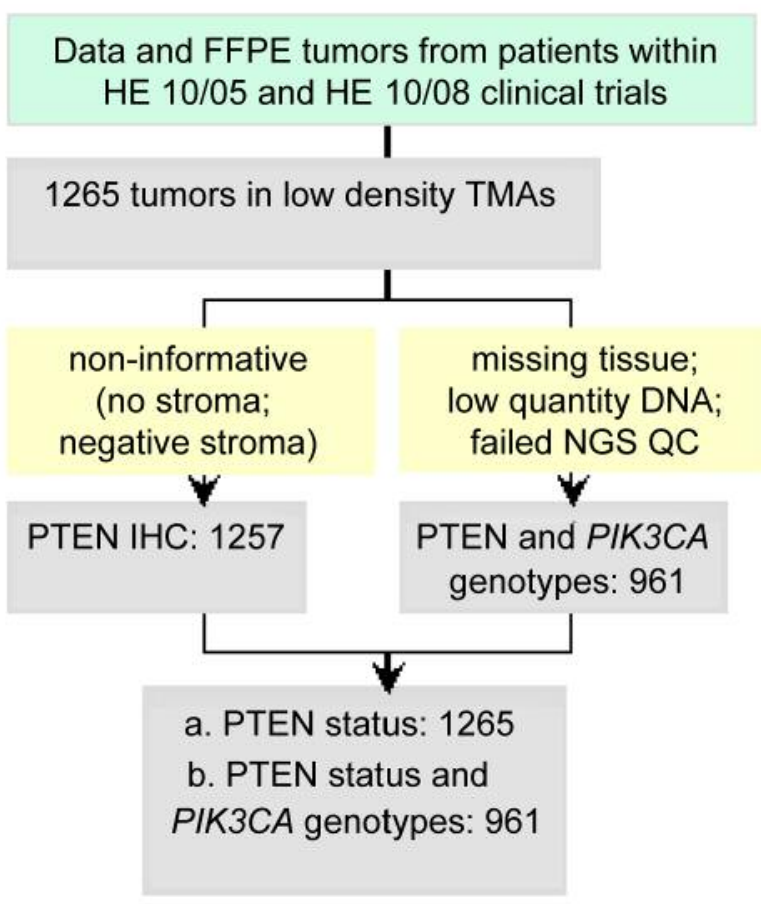

Figure 1. REMARK diagram. TMA: Tissue microarray; NGS: next generation sequencing; QC: quality control; IHC: immunohistochemistry. The final PTEN status corresponded to PTEN-loss by IHC and/or PTEN mutant; and, PTEN-no-loss by IHC only. This variable was assessed in 1265 tumors.

assessed subtypes, PIK3CA mutations and PTEN status (model 1) and the combined PTEN status/PIK3CA variable (model 2 ). The final models included variables remaining significant upon a backward selection process with a removal criterion of 0.15 . All analyses were performed in the entire cohort and in patients with concordant local/central HER2-positive tumor status.

The statistical analyses were performed using the SAS software (SAS for Windows, version 9.3, SAS Institute Inc., Cary, NC, USA). Statistical significance was set at 2 -sided $p=0.05$. Results of this study are presented according to reporting recommendations for tumor marker prognostic studies (30). This study is prospectiveretrospective (31).

\section{Results}

Clinicopathological and treatment characteristics for the 1,265 patients in the entire cohort and in each trial are presented in Table I. Most patients were $>50$ y.o. and postmenopausal; the majority had low nodal burden disease and medium sized tumors, most of which were high-grade ductal carcinomas of non-specific type; $70.4 \%$ of the tumors were ER/PgR positive and $20.6 \%$ were HER2-positive.

Among the 1,257 tumors with informative PTEN IHC, PTEN-loss was classified in 415 (33\%) with the binary Approach A and in 272 (21.6\%) with the multi-scale 

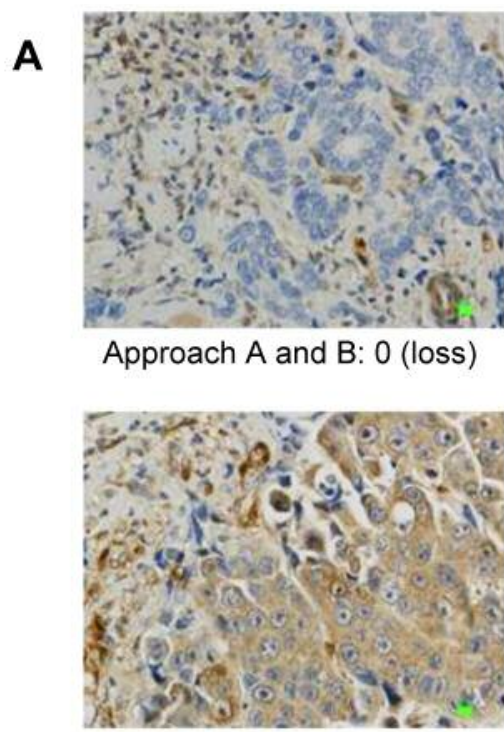

Approach A: no loss

Approach B: 1+ (no loss)

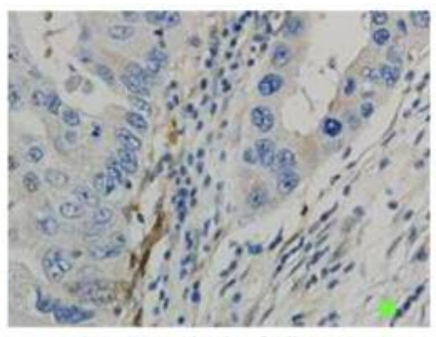

Approach A: 0 (loss)

Approach B: $1^{*}$ (no loss)

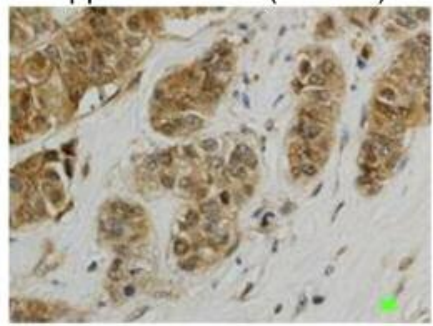

Approach A: no loss

Approach B: 2+ (no loss)

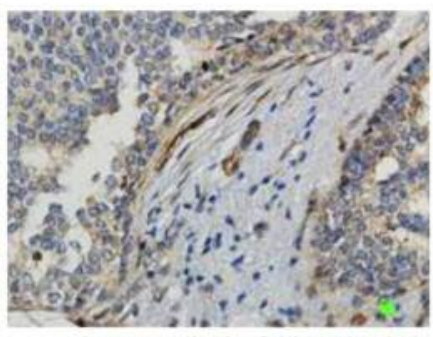

Approach A: 0 (loss)

Approach B: $1+$ (no loss)

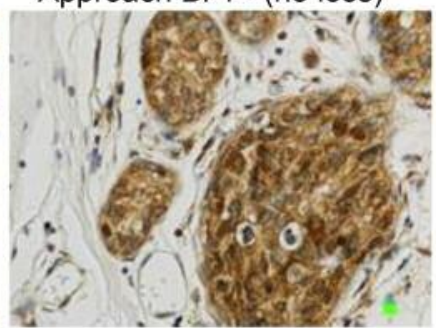

Approach A: no loss

Approach B: $3+$ (no loss)
B

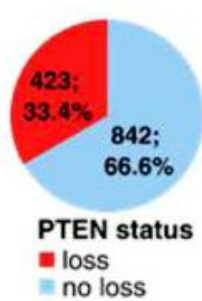

D

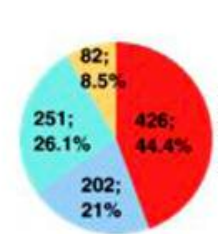

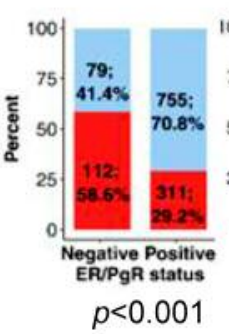
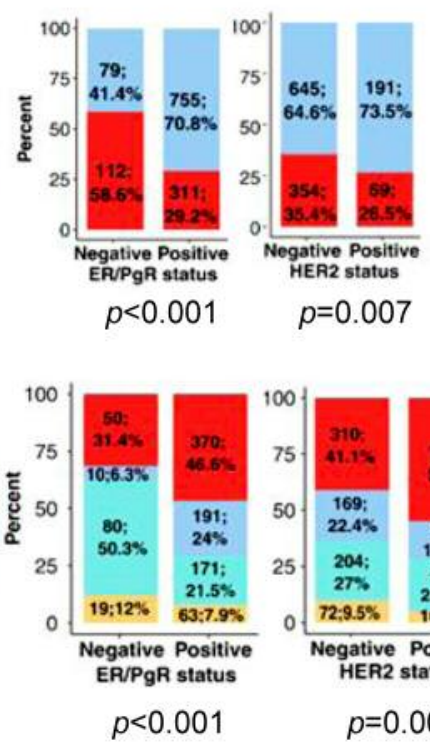
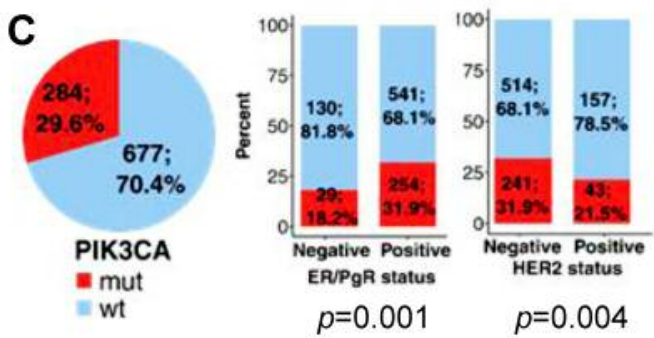

Figure 2. PTEN-loss and PIK3CA mutations in early high-risk breast tumors. A. Examples of PTEN immunohistochemistry (IHC) and evaluation of PTEN protein expression with the two approaches used in the present study. Discrepancies were observed for tumors expressing very low PTEN (pink star) or lower than the adjacent stroma and heterogeneous (green star). Green bars within microphotographs: $10 \mu \mathrm{m}$ (original magnification: X400). Note that in tumors with $2+$ and $3+$ PTEN staining intensity, it is impossible to separately evaluate cytoplasmic and nuclear staining. B. Incidence of PTEN loss in the entire cohort and its association with tumor ER and HER2 status. C. Incidence of PIK3CA mutations, as in B. D. Combined PTEN/PIK3CA alterations in the entire cohort and their associations with ER and HER2 status. approximately 1 in 4 tumors with PTENloss having PIK3CA mutations, while 1 in 3 tumors with PIK3CA mutations had PTEN-loss. In B and D: PTEN status assessed by IHC in combination with pathogenic mutations in the gene. IHC Approach A.

Approach B. Concordance between the two approaches was $84.8 \%$ with a moderate agreement (Cohen's kappa 0.624). Discordance concerned tumors with low or very low PTEN protein expression (Figure 2A); out of 191 discordant tumors, 85.9\% were classified as PTEN-loss with Approach A but as no PTEN-loss with Approach B $(p<0.001)$. HER2positive tumors were more frequently discordant than HER2negative $(p=0.010)$; again, PTEN discordance concerned 
low/very low PTEN expression in 18/18 HER2-enriched and in 22/24 Luminal-HER2 cases $(p=0.005)$.

Among 961 tumors with informative genotypes, the incidence of PTEN mutations was low, as expected for breast cancer $(4,5)$, unrelated to ER/PgR/HER2 status (32); 38 (4\%) tumors had PTEN mutations most of which had previously been classified as pathogenic. PTEN mutations and PTEN IHC were mostly concordant with PTEN IHC Approach A $(73.3 \%$ of the cases; $p<0.001)$. We used the additional 8 pathogenic mutations to characterize PTEN-loss in cases without an informative IHC. The resulting PTEN status, informative for 1265 patients, was analyzed separately with the two IHC approaches (Figure 2B for Approach A).

PIK3CA mutations were present in 284/961 (29.6\%) informative tumors (Figure 2C) and concerned missense changes in known hot-spots in exons 9 and mostly 20, as previously described $(24,25)$. PIK3CA mutations were present at higher frequencies (mean $\mathrm{VAF}=33.6 \%$ ) compared to PTEN mutations (mean $\mathrm{VAF}=22.9 \% ; p<0.001$ ), in line with the reported clonal presence and driver role of $P I K 3 C A$ mutations in breast cancer $(5,33)$.

Associations of PTEN with PIK3CA status and clinicopathologic parameters. PTEN-loss was significantly more frequent in ER/PgR-negative (58.6\%) and in HER2-negative (50.6\%) tumors (Figure 2B), while PIK3CA mutations showed the opposite distribution compared to PTEN-loss with respect to ER/PgR and HER2 status (Figure 2C). PTEN-loss was not mutually exclusive with PIK3CA mutations, although these two alterations did not tend to occur simultaneously $(p=0.020)$. Among the 961 tumors informative for PTEN status and PIK3CA genotypes, $8.5 \%$ had simultaneous PTEN-loss and PIK3CA mutations, 26.1\% had PTEN-loss only (single PTEN-loss), 21\% PIK3CA mutations only (single PIK3CA), and the rest had none of these alterations (Figure 2D).

PTEN-loss was present in more than $50 \%$ of TNBC, in about $20 \%$ of HER2-enriched tumors, and at significantly lower rates in all luminal subtypes $(p<0.001)$, while PIK3CA mutations were more frequent in Luminal $A$ and less commonly observed in HER2-enriched tumors $(p=0.001)$. PTEN-loss was associated with higher grade $(p<0.001)$, higher TILs density $(p=0.011)$ and higher proliferation rate $(p=0.002)$, in line with its prevalence in ER/PgR-negative tumors. By contrast, PIK3CA mutations were associated with lower grade $(p<0.001)$, marginally lower TILs density $(p=0.058)$ and lower proliferation rate $(p<0.001)$, in line with their prevalence in ER/PgR-positive disease. The above described characteristics of PTEN-loss were exaggerated for tumors with single PTEN-loss. PTEN-loss \& PIK3CA mutations rarely coexisted in the HER2-positive subtypes, while single $P I K 3 C A$ mutations were infrequent in TNBC $(p<0.001)$. PTEN-loss/PIK3CA-mutation patterns were similar with the multi-scale IHC Approach B.
PTEN-loss and PIK3CA mutations on patient outcome. In the entire cohort of 1265 patients, $174(13.8 \%)$ relapses and 102 $(8.1 \%)$ deaths were recorded; at a median follow-up of 64.8 months (mean=62.2 months, range=2-90 months) median DFS and OS had not been reached. Patients who had breast conserving surgery, received adjuvant hormonotherapy, had lower nodal burden and tumors of lower size, lower grade, and lower proliferation rate suffered less relapses and deaths compared to the opposite conditions.

In the entire cohort and upon univariate analysis, patients with PTEN-loss fared significantly worse than patients with preserved PTEN protein expression (Figure 3, A and B) exhibiting higher risk for relapse and death (DFS: $\mathrm{HR}=1.65$, $95 \% \mathrm{CI}=1.22-2.23$, Wald's $p=0.001$; OS: $\mathrm{HR}=2.12,95 \% \mathrm{CI}=$ 1.44-3.12, $p<0.001)$. PIK3CA mutations were not associated with relapse and death. Single PTEN-loss was associated with significantly more relapses and deaths compared to the "normal" condition for these two molecules (no PTEN-loss, no PIK3CA mutations) and to single PIK3CA-mutations (Figure $3 \mathrm{C}$ and $\mathrm{D}$ ); patients with tumors exhibiting both PTEN-loss and PIK3CA mutations had similar outcomes as those without these alterations.

In patients with HER2-positive tumors, the above statistical significance of unfavorable PTEN-loss was not retained but the overall effect of PTEN-loss was in the same direction after the 3rd year (Figure 3, E and F). Preservation of the trends for single unfavorable PTEN-loss and favorable PIK3CA mutations was noticed, but the numbers in the categories of this combined variable were partially very small for reliable comparisons.

Upon adjusting for all the significant clinicopathological variables described above, PTEN-loss and single PTEN-loss retained their unfavorable prognostic significance in the entire cohort for both DFS and OS, while single PIK3CAmutations remained as an independent favorable prognostic factor (Figure 4). Except for the clinicopathological variables, all of which retained their independent prognostic significance, increased TILs density was also an independent favorable prognosticator in the entire cohort. In patients with HER2-positive tumors, increased TILs density was an independent favorable prognostic parameter for both DFS and OS, while PTEN-loss exhibited a non-significant trend as an unfavorable prognosticator.

All the above results with respect to PTEN-loss were obtained with the binary IHC Approach A. Similar results were also obtained in the entire cohort with the multi-scale Approach B upon log-rank testing. With respect to HER2positive disease, IHC method discrepancy resulted in less than half patients with PTEN-loss, when assessed with Approach B. The outcome of these patients was apparently not different compared to HER2-positives without PTENloss. An independent unfavorable prognostic effect was yielded for PTEN-loss upon multivariate analysis; however, 


\section{A}

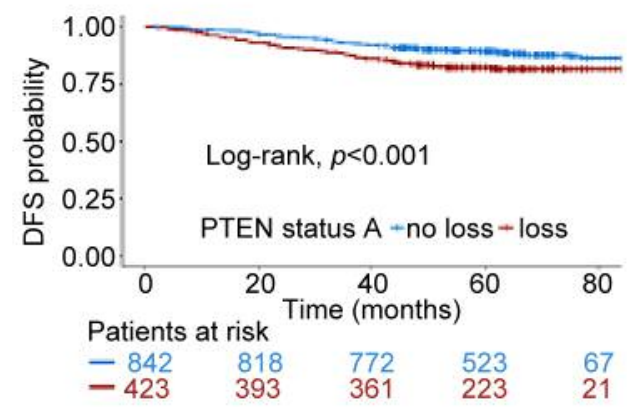

C

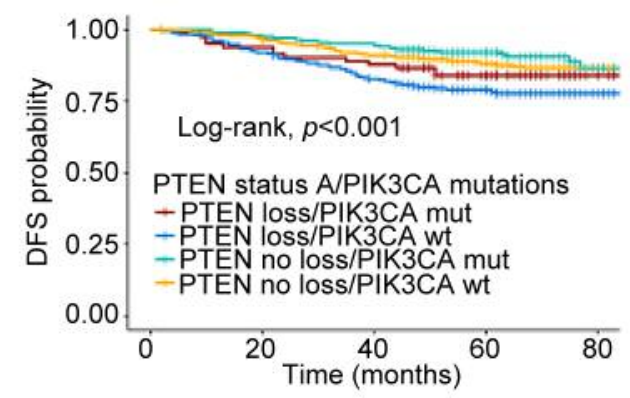

Patients at risk

\begin{tabular}{ccccc}
-82 & 77 & 72 & 45 & 5 \\
-251 & 230 & 205 & 135 & 16 \\
-202 & 198 & 192 & 147 & 19 \\
\hline
\end{tabular}

E

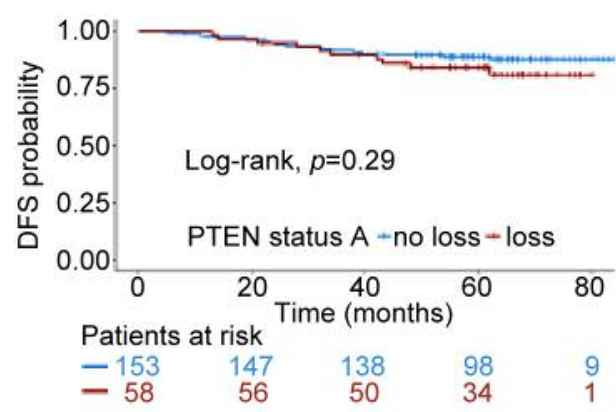

B

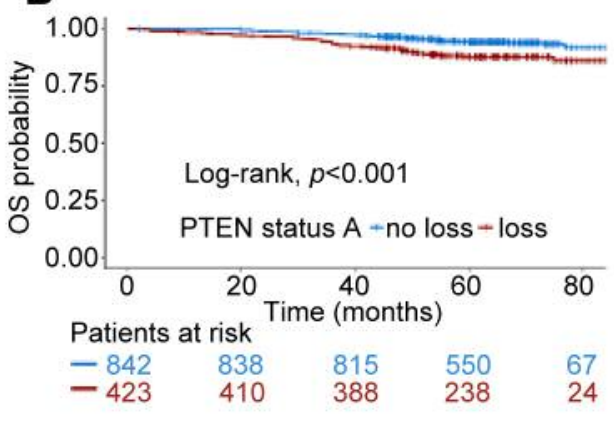

D

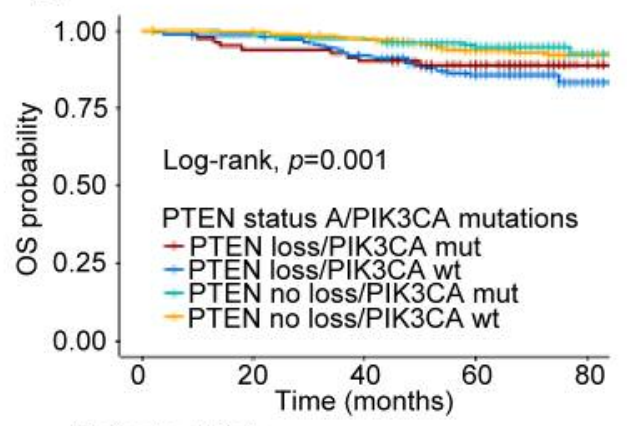

Patients at risk

$\begin{array}{ccccc}-82 & 77 & 74 & 48 & 6 \\ -251 & 246 & 228 & 147 & 18 \\ -202 & 201 & 197 & 151 & 19 \\ -426 & 424 & 411 & 288 & 48\end{array}$

$\mathbf{F}$

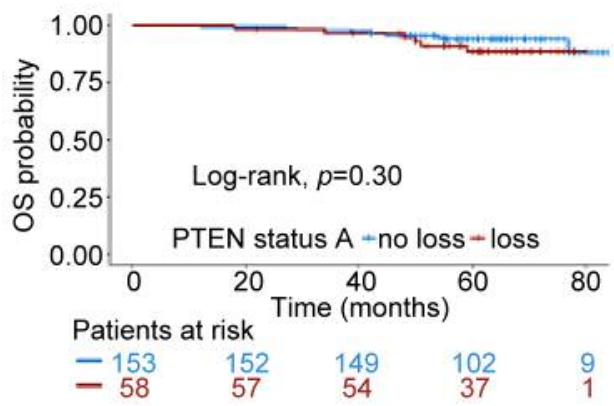

Figure 3. Association of PTEN-loss with patient disease-free (DFS) and overall survival (OS). A \& B: PTEN status in the entire cohort. C \& D: Combined PTEN/PIK3CA alterations in the entire cohort. E \& F: PTEN status in HER2-positive patients. All panels: PTEN status evaluated with the binary IHC Approach A.

the corresponding group of HER2-positive tumors with PTEN-loss was too small for statistical reliability.

\section{Discussion}

Approximately $55 \%$ of early high-risk breast cancers in the present series demonstrated PTEN-loss and/or PIK3CA mutations, as generally reported $(1,19)$. Out of the two markers, PTEN-loss was independently associated with an aggravated outcome in patients treated with adjuvant chemotherapy, in line with a meta-analysis on the adverse impact of PTEN-loss in all stages of breast cancer (15). The novel finding here is that, when present singly, PTEN-loss and PIK3CA mutations are associated with opposite prognosis. The opposite impact of single PTEN-loss and PIK3CA mutations was fully compatible with the observed 


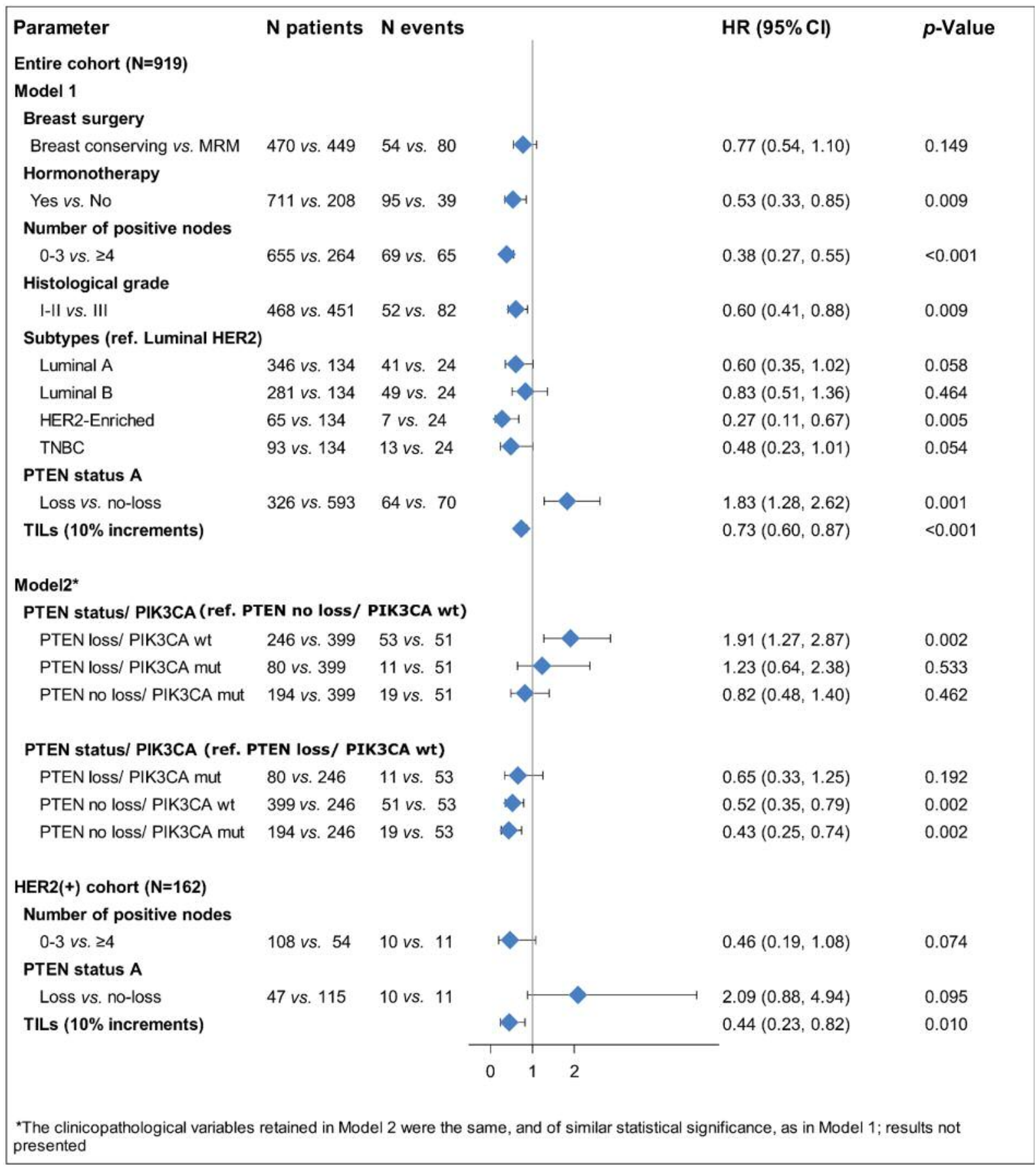

Figure 4. Forest plot demonstrating the impact of PTEN status on DFS, alone and in combination with PIK3CA status in the entire cohort and in HER2-positive patients. PTEN status evaluated with the binary IHC Approach A.

associations of these markers with clinicopathological characteristics. In comparison, in the relatively few tumors with co-altered PTEN and PIK3CA, the unfavourable effect of PTEN prevailed in the first years, indicating an inverse interaction between PTEN-loss and PIK3CA mutations. This finding is related to our previous observation in a different cohort of patients treated in the pre-trastuzumab era (16), where PIK3CA mutation types interacted with PTEN status and affected outcome in opposite directions. Further in breast cancer, TP53 mutation status may influence the prognostic 
effect of PIK3CA mutations (25) and of preserved PTEN (34), while the unfavourable effect of PTEN-loss may be augmented in the presence of additional alterations interfering with the activation of the PI3K pathway (35). All this argumentation suggests that we need to shift our view from single oncogenic drivers to a molecular environment that certainly interferes with- and may alter the effects of these drivers (36). PTEN and PIK3CA alterations are considered as oncogenic drivers within the same pathway but may counteract each other, e.g., in experimental models investigating sensitivity to mTOR inhibitors (37). The opposing effects of single PTEN-loss and PIK3CA mutations further support concerns that these two alterations may be inadequate as markers of PI3K activation for selecting patients to receive inhibitors of this pathway $(19,38,39)$. Our finding is important to consider when designing clinical trials for testing PI3K inhibitors, where PIK3CA mutations and PTEN-loss may need to be co-evaluated.

PTEN-loss as a marker suffers from method and assay standardization, which has mainly prevented its inclusion in the last ASCO recommendations for markers in the adjuvant setting in breast cancer (40). Here, we applied two methodological approaches for the evaluation of PTEN IHC: one, upfront binary (loss/no-loss), similar to most previously reported studies dealing with PTEN-loss $(14,17)$ including our own $(16,41)$; the other, initially multi-scale, was based on the intensity of PTEN protein expression in the tumor stroma (11). We found that the difference between the two methods for the final evaluation of PTEN-loss is due (i) to tumors with low or very low PTEN protein expression, and (ii) to intra-tumoral PTEN heterogeneity, which is a novel finding. For the first point, low protein expression, especially when classification is based on staining intensity, is a known issue for calling a marker positive or negative with IHC. Such "grey zone" tumors that switch class from PTEN-noloss to PTEN-loss or from PTEN-high to PTEN-low according to the method used for evaluation, as previously reported (17), constituted about $15 \%$ of all tumors in the present series. Particularly in breast cancer, given that even subtle differences in PTEN expression may be important for its biological implications (6); PTEN-loss is often used for PTEN-low although these are biologically distinct (11); genomic PTEN alterations underlying PTEN-loss or low expression are infrequent (5) or may be the result of mutational processes unrelated to PTEN itself (32); it appears doubtful that PTEN status assessed with IHC will ever be appreciated as a marker of clinical value.

Further complicating PTEN-loss as a potential marker for clinical evaluation is its heterogeneous state in primary breast tumors. We observed heterogeneous PTEN-loss in $8-13 \%$ of tumors according to the method used, although the prevalence of this condition might actually be higher since we only used TMA cores in the present study. Intra-tumoral heterogeneity mostly concerned "grey zone" tumors and may be attributed to non-genomic PTEN alterations in breast cancer $(4,5,32)$. However, it may also reflect the presence of different subclones with and without genomic PTEN alterations in the same tumor, as we show here in the limited subset of tumors with PTEN mutations that were mostly represented in tumor subpopulations. Subclonal PTEN alterations in primary tumors may become overt in metastases and result in treatment failure (42). As a method, IHC may aid in assessing PTEN status heterogeneity but again, heterogeneous tumors can be classified as PTEN-loss or as no-loss with equally sound argumentation.

The incidence of PTEN-loss in the present series, 33\% and $22 \%$ according to the approach used for IHC evaluation, lies within the previously described range for this alteration in breast cancer, e.g., $21 \%$ and $46 \%$ (11), 37\% (43), $48 \%$ (14). With respect to breast cancer subtypes, PTEN-loss is reported as a feature of ER/PgR negative and HER2 negative disease, concerning mainly TNBC $(11,13,32)$, unlike PIK3CA mutations that are characteristic for ER-positive disease (5, $24,25,33,44)$. The presented patterns of PTEN-loss and PIK3CA mutations are in line with these reports, but we also observed a $20 \%$ incidence of PTEN-loss in HER2-enriched tumors (HER2-positive/ER-negative) compared to less than $10 \%$ in luminal-HER2 (triple positive), which is in line with the notion that luminal-HER2 is more an ER-driven than a HER2-driven disease (45). PTEN-loss is reported with variable incidence in HER2-positive disease (11, 17, 44), which may reflect issues in terminology concerning PTENloss vs. PTEN-low (11); methodology, concerning modes of IHC evaluation (17) as shown here as well; and, biology, since complete PTEN-loss may not be biologically relevant in a molecular environment dominated by at least one oncogenic driver, such as HER2 over-expression (32). Our results on a high incidence of PTEN "grey zone" and heterogeneous tumors in HER2-positive disease are in line with these literature data and may explain why PTEN-loss did not significantly impact the outcome of HER2-positive cancer treated in the adjuvant setting with chemotherapy and trastuzumab, as previously observed $(11,17,44)$.

In conclusion, in early high-risk breast cancer, PTEN-loss is an unfavourable prognosticator, while single PTEN-loss and PIK3CA mutations may confer opposite effects on patient outcome. This finding has implications in the design of trials testing for PI3K-inhibitors. We also demonstrated that the method used for the evaluation of PTEN status with IHC and the heterogeneity of PTEN expression within the same tumor affect the classification of PTEN-loss and therefore, the statistical power of the performed analyses and the significance of this marker. These methodology issues are particularly important for the evaluation of PTEN status in HER2-positive disease exhibiting low rates of PTEN-loss, but high rates of PTEN "grey zone" and heterogeneity. Redirecting our efforts 
towards the search of contributors to PTEN inactivation, despite its low expression, may prove more useful for identifying intra-cellular markers of trastuzumab resistance and sensitivity to PI3K-inhibitors.

\section{Funding}

This study was supported by a Hellenic Society for Medical Oncology (HeSMO) grant and by an internal Hellenic Cooperative Oncology Group (HeCOG) translational research grant (HE TRANS_BR). The funders played no role in study design, data collection and analysis, decision to publish or preparation of the manuscript.

\section{Conflicts of Interest}

G. Lazaridis: Remuneration: Lecture Honoraria by BMS, MSD, Roche, Amgen, LEO Pharma. Consultation/Advisory role: Merck. Funding: Scholarship by HeSMO (Hellenic Society of Medical Oncology); C. Christodoulou: Consultation/Advisory role: Roche, Genesis, Pfizer, Novartis, MSD, BMS, Astra-Zeneca, Eli Lilly; H. Gogas: Funding: Novartis, Roche (Institution grant); G. Fountzilas: Remuneration: Honoraria by Astra-Zeneca. Consultation/Advisory role: Pfizer, Sanofi, Roche; The rest of the Authors declare no conflict of interest.

\section{Authors' Contributions}

Study conception: GL. VK, GF; Study Design: GL, VK, IK, ET, GF; Acquisition of data: GL, EV, MB, MS, GP, EE, APB, AP, CC, HG, AK, ET, DP, FZ, GF; Analysis and interpretation: GL, VK, EV, KM, KP, EG, MB, ET, GF; Drafted the manuscript: GL, VK, IK, KM, KP, EG, Et, GE. All Authors have revised and approved the manuscript.

\section{Acknowledgements}

The Authors are indebted to all patients and their families for their trust and participation in the Hellenic Cooperative Oncology Group trials and for the provision of biological material for research purposes. The Authors wish to thank Eneida Jaupaj for tissue samples collection and Maria Moschoni for data coordination.

\section{References}

1 Paplomata E and O'Regan R: The PI3K/AKT/mTOR pathway in breast cancer: Targets, trials and biomarkers. Ther Adv Med Oncol 6(4): 154-166, 2014. PMID: 25057302. DOI: 10.1177/ 1758834014530023

2 Milella M, Falcone I, Conciatori F, Cesta Incani U, Del Curatolo A, Inzerilli N, Nuzzo CM, Vaccaro V, Vari S, Cognetti F and Ciuffreda L: Pten: Multiple functions in human malignant tumors. Front Oncol 5: 24, 2015. PMID: 25763354. DOI: $10.3389 /$ fonc 2015.00024

3 Bowen KA, Doan HQ, Zhou BP, Wang Q, Zhou Y, Rychahou PG and Evers BM: PTEN loss induces epithelial--mesenchymal transition in human colon cancer cells. Anticancer Res 29(11): 4439-4449, 2009. PMID: 20032390.
4 Hollander MC, Blumenthal GM and Dennis PA: PTEN loss in the continuum of common cancers, rare syndromes and mouse models. Nature Reviews Cancer 11(289): 2011. PMID: 21430697. DOI: $10.1038 / \mathrm{nrc} 3037$

5 Comprehensive molecular portraits of human breast tumours. Nature 490(7418): 61-70, 2012. PMID: 23000897.

6 Alimonti A, Carracedo A, Clohessy JG, Trotman LC, Nardella C, Egia A, Salmena L, Sampieri K, Haveman WJ, Brogi E, Richardson AL, Zhang J and Pandolfi PP: Subtle variations in PTEN dose determine cancer susceptibility. Nat Genet 42(5): 454-458, 2010. PMID: 20400965. DOI: $10.1038 / n g .556$

7 Zhang XC, Piccini A, Myers MP, Van Aelst L and Tonks NK: Functional analysis of the protein phosphatase activity of PTEN. Biochem J 444(3): 457-464, 2012. PMID: 22413754. DOI: 10.1042/bj20120098

8 Kechagioglou P, Papi RM, Provatopoulou X, Kalogera E, Papadimitriou E, Grigoropoulos P, Nonni A, Zografos G, Kyriakidis DA and Gounaris A: Tumor suppressor PTEN in breast cancer: Heterozygosity, mutations and protein expression. Anticancer Res 34(3): 1387-1400, 2014. PMID: 24596386.

9 Noh WC, Kim YH, Kim MS, Koh JS, Kim HA, Moon NM and Paik NS: Activation of the mtor signaling pathway in breast cancer and its correlation with the clinicopathologic variables. Breast Cancer Res Treat 110(3): 477-483, 2008. PMID: 17805960. DOI: 10.1007/s10549-007-9746-x

10 Iqbal J, Thike AA, Cheok PY, Tse GM and Tan PH: Insulin growth factor receptor-1 expression and loss of PTEN protein predict early recurrence in triple-negative breast cancer. Histopathology 61(4): 652-659, 2012. PMID: 22759273. DOI: 10.1111/j.1365-2559.2012.04255.x

11 Stern HM, Gardner H, Burzykowski T, Elatre W, O'Brien C, Lackner MR, Pestano GA, Santiago A, Villalobos I, Eiermann W, Pienkowski T, Martin M, Robert N, Crown J, Nuciforo P, Bee V, Mackey J, Slamon DJ and Press MF: PTEN loss is associated with worse outcome in her2-amplified breast cancer patients but is not associated with trastuzumab resistance. Clin Cancer Res 21(9): 2065-2074, 2015. PMID: 25649019. DOI: 10.1158/1078-0432.ccr-14-2993

12 Bianchini G, Kiermaier A, Bianchi GV, Im YH, Pienkowski T, Liu MC, Tseng LM, Dowsett M, Zabaglo L, Kirk S, Szado T, Eng-Wong J, Amler LC, Valagussa P and Gianni L: Biomarker analysis of the neosphere study: Pertuzumab, trastuzumab, and docetaxel versus trastuzumab plus docetaxel, pertuzumab plus trastuzumab, or pertuzumab plus docetaxel for the neoadjuvant treatment of her2-positive breast cancer. Breast Cancer Res 19(1): 16, 2017. PMID: 28183321. DOI: 10.1186/s13058-0170806-9

13 Li S, Shen Y, Wang M, Yang J, Lv M, Li P, Chen Z and Yang J: Loss of PTEN expression in breast cancer: Association with clinicopathological characteristics and prognosis. Oncotarget 8(19): 32043-32054, 2017. PMID: 5458267. DOI: 10.18632/ oncotarget.16761

14 Depowski PL, Rosenthal SI and Ross JS: Loss of expression of the PTEN gene protein product is associated with poor outcome in breast cancer. Mod Pathol 14(7): 672-676, 2001. PMID: 11454999. DOI:10.1038/modpathol.3880371

15 Yang ZY, Yu YY, Yuan JQ, Shen WX, Zheng DY, Chen JZ, Mao $\mathrm{C}$ and Tang JL: The prognostic value of phosphatase and tensin homolog negativity in breast cancer: A systematic review and meta-analysis of 32 studies with 4393 patients. Crit Rev Oncol 
Hematol 101: 40-49, 2016. PMID: 26951995. DOI: 10.1016/ j.critrevonc.2016.01.013

16 Papaxoinis G, Kotoula V, Alexopoulou Z, Kalogeras KT, Zagouri F, Timotheadou E, Gogas H, Pentheroudakis G, Christodoulou C, Koutras A, Bafaloukos D, Aravantinos G, Papakostas P, Charalambous E, Papadopoulou K, Varthalitis I, Efstratiou I, Zaramboukas T, Patsea H, Scopa CD, Skondra M, Kosmidis P, Pectasides D and Fountzilas G: Significance of pik3ca mutations in patients with early breast cancer treated with adjuvant chemotherapy: A hellenic cooperative oncology group (hecog) study. PLoS One 10(10): e0140293, 2015. PMID: 26452060. DOI: 10.1371/journal.pone.0140293

17 Perez EA, Dueck AC, McCullough AE, Chen B, Geiger XJ, Jenkins RB, Lingle WL, Davidson NE, Martino S, Kaufman PA, Kutteh LA, Sledge GW, Harris LN, Gralow JR and Reinholz MM: Impact of PTEN protein expression on benefit from adjuvant trastuzumab in early-stage human epidermal growth factor receptor 2-positive breast cancer in the north central cancer treatment group n9831 trial. J Clin Oncol 31(17): 21152122, 2013. PMID: 3731983. DOI: 10.1200/JCO.2012.42.2642

18 Mayer I: Role of mTOR inhibition in preventing resistance and restoring sensitivity to hormone-targeted and her2-targeted therapies in breast cancer. Clin Adv Hematol Oncol 11(4): 217224, 2013. PMID: 3774138.

19 Janku F, Yap TA and Meric-Bernstam F: Targeting the pi3k pathway in cancer: Are we making headway? Nat Rev Clin Oncol 15: 273, 2018. PMID: 29508857. DOI: 10.1038/ nrclinonc. 2018.28

20 Nagata Y, Lan KH, Zhou X, Tan M, Esteva FJ, Sahin AA, Klos KS, Li P, Monia BP, Nguyen NT, Hortobagyi GN, Hung MC and Yu D: PTEN activation contributes to tumor inhibition by trastuzumab, and loss of PTEN predicts trastuzumab resistance in patients. Cancer Cell 6(2): 117-127, 2004. PMID: 15324695. DOI: $10.1016 /$ j.ccr.2004.06.022

21 Nuciforo PG, Aura C, Holmes E, Prudkin L, Jimenez J, Martinez P, Ameels H, de la Pena L, Ellis C, Eidtmann H, Piccart-Gebhart MJ, Scaltriti $M$ and Baselga $J$ : Benefit to neoadjuvant antihuman epidermal growth factor receptor 2 (her2)-targeted therapies in HER2-positive primary breast cancer is independent of phosphatase and tensin homolog deleted from chromosome 10 (PTEN) status. Ann Oncol 26(7): 1494-1500, 2015. PMID: 25851628. DOI: $10.1093 / \mathrm{annonc} / \mathrm{mdv} 175$

22 Rimawi MF, De Angelis C, Contreras A, Pareja F, Geyer FC, Burke KA, Herrera S, Wang T, Mayer IA, Forero A, Nanda R, Goetz MP, Chang JC, Krop IE, Wolff AC, Pavlick AC, Fuqua SAW, Gutierrez C, Hilsenbeck SG, Li MM, Weigelt B, ReisFilho JS, Kent Osborne C and Schiff R: Low PTEN levels and pik3ca mutations predict resistance to neoadjuvant lapatinib and trastuzumab without chemotherapy in patients with HER2 overexpressing breast cancer. Breast Cancer Res Treat 167(3): 731740, 2018. PMID: 5821069. DOI: 10.1007/s10549-017-4533-9

23 Fountzilas G, Dafni U, Papadimitriou C, Timotheadou E, Gogas H, Eleftheraki AG, Xanthakis I, Christodoulou C, Koutras A, Papandreou CN, Papakostas P, Miliaras S, Markopoulos C, Dimitrakakis C, Korantzopoulos P, Karanikiotis C, Bafaloukos D, Kosmidis P, Samantas E, Varthalitis I, Pavlidis N, Pectasides $\mathrm{D}$ and Dimopoulos MA: Dose-dense sequential adjuvant chemotherapy followed, as indicated, by trastuzumab for one year in patients with early breast cancer: First report at 5-year median follow-up of a hellenic cooperative oncology group randomized phase iii trial. BMC Cancer 14: 515, 2014. PMID: 25026897. DOI: $10.1186 / 1471-2407-14-515$

24 Fountzilas G, Giannoulatou E, Alexopoulou Z, Zagouri F, Timotheadou E, Papadopoulou K, Lakis S, Bobos M, Poulios C, Sotiropoulou M, Lyberopoulou A, Gogas H, Pentheroudakis G, Pectasides D, Koutras A, Christodoulou C, Papandreou C, Samantas E, Papakostas P, Kosmidis P, Bafaloukos D, Karanikiotis C, Dimopoulos MA and Kotoula V: TP53 mutations and protein immunopositivity may predict for poor outcome but also for trastuzumab benefit in patients with early breast cancer treated in the adjuvant setting. Oncotarget, 2016. PMID: 27129168. DOI: $10.18632 /$ oncotarget.9022

25 Kotoula V, Karavasilis V, Zagouri F, Kouvatseas G, Giannoulatou E, Gogas H, Lakis S, Pentheroudakis G, Bobos M, Papadopoulou K, Tsolaki E, Pectasides D, Lazaridis G, Koutras A, Aravantinos G, Christodoulou C, Papakostas P, Markopoulos C, Zografos G, Papandreou C and Fountzilas G: Effects of TP53 and PIK3CA mutations in early breast cancer: A matter of comutation and tumor-infiltrating lymphocytes. Breast Cancer Res Treat 158(2): 307-321, 2016. PMID: 27369359. DOI: 10.1007/ s10549-016-3883-z

26 Salgado R, Denkert C, Demaria S, Sirtaine N, Klauschen F, Pruneri G, Wienert S, Van den Eynden G, Baehner FL, PenaultLlorca F, Perez EA, Thompson EA, Symmans WF, Richardson AL, Brock J, Criscitiello C, Bailey H, Ignatiadis M, Floris G, Sparano J, Kos Z, Nielsen T, Rimm DL, Allison KH, Reis-Filho JS, Loibl S, Sotiriou C, Viale G, Badve S, Adams S, WillardGallo K, Loi S and International TWG: The evaluation of tumorinfiltrating lymphocytes (tils) in breast cancer: Recommendations by an international tils working group 2014. Ann Oncol 26(2): 259-271, 2015. PMID: 25214542. DOI: 10.1093/annonc/mdu450

27 Fountzilas G, Dafni U, Bobos M, Batistatou A, Kotoula V, Trihia H, Malamou-Mitsi V, Miliaras S, Chrisafi S, Papadopoulos S, Sotiropoulou M, Filippidis T, Gogas H, Koletsa T, Bafaloukos D, Televantou D, Kalogeras KT, Pectasides D, Skarlos DV, Koutras A and Dimopoulos MA: Differential response of immunohistochemically defined breast cancer subtypes to anthracycline-based adjuvant chemotherapy with or without paclitaxel. PLoS One 7(6): e37946, 2012. PMID: 3367950. DOI: 10.1371/journal.pone.0037946

28 Kotoula V, Lyberopoulou A, Papadopoulou K, Charalambous E, Alexopoulou Z, Gakou C, Lakis S, Tsolaki E, Lilakos K and Fountzilas G: Evaluation of two highly-multiplexed custom panels for massively parallel semiconductor sequencing on paraffin DNA. PLoS One 10(6): e0128818, 2015. PMID: 26039550. DOI: 10.1371/journal.pone.0128818

29 McGranahan N, Favero F, de Bruin EC, Birkbak NJ, Szallasi Z and Swanton C: Clonal status of actionable driver events and the timing of mutational processes in cancer evolution. Sci Transl Med 7(283): 283ra254, 2015. PMID: 4636056. DOI: 10.1126/ scitranslmed.aaa1408

30 McShane LM, Altman DG, Sauerbrei W, Taube SE, Gion M, Clark GM and Statistics Subcommittee of NCIEWGoCD: Reporting recommendations for tumor marker prognostic studies (remark). Breast Cancer Res Treat 100(2): 229-235, 2006. PMID: 16932852. DOI: 10.1007/s10549-006-9242-8

31 Simon RM, Paik S and Hayes DF: Use of archived specimens in evaluation of prognostic and predictive biomarkers. J Natl Cancer Inst 101(21): 1446-1452, 2009. PMID: 2782246. 
32 Jones N, Bonnet F, Sfar S, Lafitte M, Lafon D, Sierankowski G, Brouste V, Banneau G, Tunon de Lara C, Debled M, MacGrogan G, Longy M and Sevenet N: Comprehensive analysis of PTEN status in breast carcinomas. Int J Cancer 133(2): 323-334, 2013. PMID: 23319441. DOI: 10.1002/ijc.28021

33 Stephens PJ, Tarpey PS, Davies H, Van Loo P, Greenman C, Wedge DC, Nik-Zainal S, Martin S, Varela I, Bignell GR, Yates LR, Papaemmanuil E, Beare D, Butler A, Cheverton A, Gamble J, Hinton J, Jia M, Jayakumar A, Jones D, Latimer C, Lau KW, McLaren S, McBride DJ, Menzies A, Mudie L, Raine K, Rad R, Chapman MS, Teague J, Easton D, Langerod A, Oslo Breast Cancer C, Lee MT, Shen CY, Tee BT, Huimin BW, Broeks A, Vargas AC, Turashvili G, Martens J, Fatima A, Miron P, Chin SF, Thomas G, Boyault S, Mariani O, Lakhani SR, van de Vijver M, van 't Veer L, Foekens J, Desmedt C, Sotiriou C, Tutt A, Caldas C, Reis-Filho JS, Aparicio SA, Salomon AV, BorresenDale AL, Richardson AL, Campbell PJ, Futreal PA and Stratton MR: The landscape of cancer genes and mutational processes in breast cancer. Nature 486(7403): 400-404, 2012. PMID: 3428862. DOI: $10.1038 /$ nature 11017

34 Yndestad S, Austreid E, Knappskog S, Chrisanthar R, Lilleng PK, Lonning PE and Eikesdal HP: High PTEN gene expression is a negative prognostic marker in human primary breast cancers with preserved p53 function. Breast Cancer Res Treat 163(1): 177-190, 2017. PMID: 5387035. DOI: 10.1007/s10549-0174160-5

35 Lebok P, Kopperschmidt V, Kluth M, Hube-Magg C, Ozden C, B T, Hussein K, Mittenzwei A, Lebeau A, Witzel I, Wolber L, Mahner S, Janicke F, Geist S, Paluchowski P, Wilke C, Heilenkotter U, Simon R, Sauter G, Terracciano L, Krech R, von d Assen A, Muller V and Burandt E: Partial PTEN deletion is linked to poor prognosis in breast cancer. BMC Cancer 15: 963, 2015. PMID: 4682275. DOI: 10.1186/s12885-015-1770-3

36 Mina M, Raynaud F, Tavernari D, Battistello E, Sungalee S, Saghafinia S, Laessle T, Sanchez-Vega F, Schultz N, Oricchio E and Ciriello G: Conditional selection of genomic alterations dictates cancer evolution and oncogenic dependencies. Cancer Cell 32(2): 155-168 e156, 2017. PMID: 28756993. DOI: 10.1016/j.ccell.2017.06.010

37 Weigelt B, Warne PH and Downward J: PIK3CA mutation, but not PTEN loss of function, determines the sensitivity of breast cancer cells to mTOR inhibitory drugs. Oncogene 30(29): 32223233, 2011. PMID: 21358673. DOI: 10.1038/onc.2011.42

38 Zardavas D, Phillips WA and Loi S: Pik3ca mutations in breast cancer: Reconciling findings from preclinical and clinical data. Breast Cancer Res 16(1): 201, 2014. PMID: 4054885. DOI: 10.1186/bcr3605

39 Ponde N, Brandao M, El-Hachem G, Werbrouck E and Piccart M: Treatment of advanced HER2-positive breast cancer: 2018 and beyond. Cancer Treat Rev 67: 10-20, 2018. PMID: 29751334. DOI: 10.1016/j.ctrv.2018.04.016
40 Harris LN, Ismaila N, McShane LM and Hayes DF: Use of biomarkers to guide decisions on adjuvant systemic therapy for women with early-stage invasive breast cancer: American society of clinical oncology clinical practice guideline summary. J Oncol Pract 12(4): 384-389, 2016. PMID: 26957642. DOI: 10.1200/jop.2016.010868

41 Razis E, Bobos M, Kotoula V, Eleftheraki AG, Kalofonos HP, Pavlakis K, Papakostas P, Aravantinos G, Rigakos G, Efstratiou I, Petraki K, Bafaloukos D, Kostopoulos I, Pectasides D, Kalogeras KT, Skarlos D and Fountzilas G: Evaluation of the association of PIK3CA mutations and loss with efficacy of trastuzumab therapy in metastatic breast cancer. Breast Cancer Res Treat 128(2): 447-456, 2011. PMID: 21594665. DOI: 10.1007/s 10549-011-1572-5

42 Juric D, Castel P, Griffith M, Griffith OL, Won HH, Ellis H, Ebbesen SH, Ainscough BJ, Ramu A, Iyer G, Shah RH, Huynh T, Mino-Kenudson M, Sgroi D, Isakoff S, Thabet A, Elamine L, Solit DB, Lowe SW, Quadt C, Peters M, Derti A, Schegel R, Huang A, Mardis ER, Berger MF, Baselga J and Scaltriti M: Convergent loss of PTEN leads to clinical resistance to a pi(3)kalpha inhibitor. Nature 518(7538): 240-244, 2015. PMID: 4326538. DOI: 10.1038 /nature 13948

43 Perez-Tenorio G, Alkhori L, Olsson B, Waltersson MA, Nordenskjold B, Rutqvist LE, Skoog L and Stal O: PIK3CA mutations and PTEN loss correlate with similar prognostic factors and are not mutually exclusive in breast cancer. Clin Cancer Res 13(12): 3577-3584, 2007. PMID: 17575221. DOI: 10.1158/1078-0432.ccr-06-1609

44 Jensen JD, Knoop A, Laenkholm AV, Grauslund M, Jensen MB, Santoni-Rugiu E, Andersson $M$ and Ewertz M: PIK3CA mutations, PTEN, and pHER2 expression and impact on outcome in HER2-positive early-stage breast cancer patients treated with adjuvant chemotherapy and trastuzumab. Ann Oncol 23(8): 2034-2042, 2012. PMID: 22172323. DOI: 10.1093/ annonc/mdr546

45 Llombart-Cussac A, Cortes J, Pare L, Galvan P, Bermejo B, Martinez N, Vidal M, Pernas S, Lopez R, Munoz M, Nuciforo P, Morales S, Oliveira M, de la Pena L, Pelaez A and Prat A: HER2-enriched subtype as a predictor of pathological complete response following trastuzumab and lapatinib without chemotherapy in early-stage HER2-positive breast cancer (PAMELA): An open-label, single-group, multicentre, phase 2 trial. Lancet Oncol 18(4): 545-554, 2017. PMID: 28238593. DOI: $10.1016 / \mathrm{S} 1470-2045(17) 30021-9$

Received February 11, 2019

Revised March 28, 2019 Accepted March 29, 2019 